Johnson \& Wales University ScholarsArchive@JWU

Health \& Wellness Department Faculty

Publications and Research

College of Health \& Wellness

$1-2018$

\title{
Perceptions of Alcohol Advertising among High Risk Drinkers
}

Jonathan K. Noel

Johnson \& Wales University - Providence, Jonathan.Noel@jwu.edu

Ziming Xuan

Thomas F. Babor

Follow this and additional works at: https://scholarsarchive.jwu.edu/health_fac

Part of the Medicine and Health Sciences Commons

\section{Repository Citation}

Noel, Jonathan K.; Xuan, Ziming; and Babor, Thomas F., "Perceptions of Alcohol Advertising among High Risk Drinkers" (2018).

Health o Wellness Department Faculty Publications and Research. 40.

https://scholarsarchive.jwu.edu/health_fac/40 


\title{
Substance Use \& Misuse
}

\section{Perceptions of Alcohol Advertising among High Risk Drinkers}

\author{
Jonathan K. Noel, Ziming Xuan \& Thomas F. Babor
}

To cite this article: Jonathan K. Noel, Ziming Xuan \& Thomas F. Babor (2018) Perceptions of Alcohol Advertising among High Risk Drinkers, Substance Use \& Misuse, 53:9, 1403-1410, DOI: 10.1080/10826084.2017.1409765

To link to this article: https://doi.org/10.1080/10826084.2017.1409765

$$
\text { 曲 Published online: } 03 \text { Jan } 2018 .
$$

Submit your article to this journal

LIII Article views: 128

View Crossmark data \lceil 


\title{
Perceptions of Alcohol Advertising among High Risk Drinkers
}

\author{
Jonathan K. Noel ${ }^{\mathrm{a}}$, Ziming Xuan ${ }^{\mathrm{b}}$, and Thomas F. Babor ${ }^{\mathrm{a}}$ \\ aDepartment of Community Medicine and Health Care, University of Connecticut School of Medicine, Farmington, Connecticut, USA; \\ ${ }^{\mathrm{b}}$ Department of Community Health Sciences, Boston University School of Public Health, Boston, Massachusetts, USA
}

\begin{abstract}
Background: Individuals who are particularly vulnerable to the influence of alcohol advertising, such as youth, need special protections, yet little research has been done to determine if other vulnerable groups exist. Secondary data analysis was conducted to determine if perceptions of alcohol advertising differ between groups based on their alcohol use and whether the definition of "vulnerable" should be expanded beyond demographic categories. Methods: Students $(n=326)$ from 2 U.S. colleges viewed 5 alcohol ads and rated them using a scale designed to detect violations of the alcohol industry's self-regulated marketing codes. Individuals with a history of excessive alcohol use, as measured by the Alcohol Use Disorders Identification Test (AUDIT), were considered potentially vulnerable to alcohol advertising and were compared against individuals without a history of excessive alcohol use. Hierarchical linear modeling was used to determine between-group differences in 4 dependent variables (ad appeal, perceived alcohol consumption, perceived excessive drinking, and perceived responsible drinking). All models were adjusted for age, race, ethnicity, sex, and parental alcohol use. Results: AUDIT risk categories were positively associated with ad appeal ( $p<0.001)$, the amount of alcohol perceived to be consumed $(p<0.01)$, and perceptions of responsible drinking $(p<0.05)$. There was no significant associated with perceptions of excessive drinking. Conclusions: Individuals with a history of heavy alcohol use perceived greater alcohol consumption in alcohol ads but perceived this consumption, which often met the definition for binge drinking, to be responsible. Stricter regulations may be needed to protect heavy alcohol users from the effects of alcohol advertising.
\end{abstract}

\section{KEYWORDS}

Alcohol; advertising; marketing; vulnerable; perception; self-regulation

\section{Introduction}

Alcohol marketing has been identified as a potentially important risk factor for alcohol use and misuse. Two systematic reviews appraised research published prior to 2008 and concluded that exposure to alcohol marketing was associated with increased drinking intentions, increased overall alcohol consumption, and earlier alcohol initiation, although the effects were modest in some studies (Anderson, de Bruijn, Angus, Gordon, \& Hastings, 2009; Smith \& Foxcroft, 2009). A third review that focused on research published since 2008, and that had not been included in the previous review, concluded alcohol marketing increases alcohol initiation and binge or hazardous drinking (Jernigan, Noel, Landon, Thornton, \& Lobstein, 2017).

In the United States (U.S.), vulnerable groups are protected from the influences of alcohol marketing through self-regulated alcohol marketing codes. The codes describe the audience composition an alcohol ad can be broadcast to and contain restrictions on the content of alcohol advertising. Alcohol producers, trade associations, and the advertising industry have created these codes for each major type of alcoholic beverage (i.e. beer, distilled spirits, and wine), and the contents of these codes have been summarized by the International Alliance for Responsible Drinking's (IARD) Guiding Principles: Self-Regulation of Marketing Communications for Beverage Alcohol (Guiding Principles) (IARD, 2011). Within IARD's Guiding Principles, individuals under the alcohol minimum legal purchase age (MLPA) and pregnant women are explicitly mentioned, and such references suggest that the alcohol industry considers these groups vulnerable to alcohol marketing practices.

However, vulnerable groups based on behavioral characteristics may also exist. For example, heavy alcohol users are known to react strongly to generic alcohol cues. Heavy drinkers may be easily distracted by alcoholrelated stimuli (Bar-Haim et al., 2007), and increased alcohol consumption has been positively associated with increased attentional biases towards alcohol cues (Cox, Pothos, \& Hosier, 2007; Field \& Cox, 2008). Furthermore, attentional biases may increase subjective alcohol cravings (Field et al., 2007). Thus, a positive feedback loop may exist among heavy alcohol users where

CONTACT Jonathan K. Noel $\otimes$ jknoel@hotmail.com Department of Community Medicine and Health Care, University of Connecticut School of Medicine, 263 Farmington Avenue, MC 6325, Farmington, CT 06030-6325, USA.

(c) 2018 Taylor \& Francis Group, LLC 
alcohol-related stimuli become more salient as cravings increase, and cravings increase as greater attention is paid to alcohol-related stimuli (Field \& Cox, 2008). Similar observations have been observed in heavy alcohol users after exposure to alcohol advertising (Dager et al., 2013; Lee, Namkoong, Lee, An, \& Lee, 2006). It is worth noting that this potential positive feedback loop remains theoretical, and research on attentional biases has been mixed. A recent literature review concluded that while attentional biases are likely larger in heavier drug (including alcohol) users than non-users, the predictive ability of attentional biases on consumptive behavior is largely mixed (Field et al., 2016).

Current self-regulated alcohol marketing codes do not include a definition of vulnerability nor do they explicitly reference heavy alcohol users as a potentially vulnerable group. Furthermore, there is little information on how alcohol ad perceptions differ between individuals who are and are not heavy alcohol users. A secondary analysis of cross-sectional data was conducted to determine if alcohol use influences perceptions of alcohol advertisements broadcast in the U.S.

It was hypothesized that heavy alcohol users would perceive alcohol ads to be more appealing and to contain greater alcohol consumption compared to non-heavy alcohol users. To the extent that heavy alcohol users are found to be differentially responsive to alcohol advertisements, this information could be used to expand the protections included in industry self-regulatory marketing codes that define permissible exposure markets and ad content for their advertising campaigns.

\section{Methods}

This study was a secondary data analysis using data previously collected on a sample of college students who were exposed to multiple alcohol ads after completing several questionnaires on demographics and alcohol use history.

\section{Data collection}

Data were collected from 326 students recruited from two Connecticut colleges in 2003 and 2004 (Babor, Xuan, \& Proctor, 2008). After providing informed consent, study participants completed a questionnaire containing questions on demographic characteristics, individual alcohol use history, and parental alcohol use history. The demographic questions included age, gender, race, and ethnicity. Individual alcohol use history was assessed using the 10-item Alcohol Use Disorders Identification Test (AUDIT) (Saunders, Aasland, Babor, de la Fuente, \& Grant, 1993). AUDIT scores were re-coded into the four AUDIT risk categories, Zones I to IV. Zone I (scores 0-7) refers to abstinence or low-risk drinking. Zone II (scores
8-15) suggests drinking levels above low-risk guidelines (i.e. $\geq 5$ drinks per session or $\geq 14$ drinks per week for men and $\geq 4$ drinks per session or $\geq 7$ drinks per week for women) (Centers for Disease Control and Prevention [CDC], 2016), while Zone III (scores 16-19) suggests a consistent pattern of hazardous alcohol consumption (Saunders et al., 1993). Zone IV (scores 20-40) suggests participants may be alcohol dependent.

Parental alcohol use history was assessed using two items. Participants were asked to describe their father's (or male guardian's) and their mother's (or female guardian's) use of alcohol during most of the time they were growing up using the following response categories: abstainer, former drinker in recovery or recovered, infrequent or light drinking, moderate drinking, heavy drinker, problem drinker, I do not know, or not applicable.

After completion of the initial questionnaire, each participant viewed five unique alcohol advertisements, four television ads, and one print ad. After each ad, participants answered 48 questions based on the 1997 U.S. Beer Institute's Marketing and Advertising code (Babor et al., 2008). Four of these questions were used as dependent variables in the analysis. One question asked about ad appeal (i.e. "How appealing is this ad to you?"), which was measured on a five point Likert scale ranging from very unappealing to very appealing. Two questions used five point Likert scales with response options ranging from strongly disagree to strongly agree. One question focused on excessive alcohol consumption (i.e. "This ad shows situations where people are drinking an alcoholic beverage excessively") and the other focused on responsible alcohol consumption (i.e. "This ad shows situations where people are drinking alcohol responsibly"). The fourth question asked about how many drinks the participant perceived the main character of the ad to be have consumed (i.e. "How many drinks do you estimate this person is likely to consume in the situation shown in the ad?"). These questions were selected because of previous research suggesting that students may perceive the characters in alcohol ads to be heavy episodic drinkers and the number of alcohol dependence symptoms was positively associated with the amount of alcohol perceived to be consumed (Proctor, Babor, \& Xuan, 2005). Moreover, heavier alcohol consumption has been associated with perceiving alcohol ads to be more appealing (Vendrame, Pinsky, Faria, \& Silva, 2009).

\section{Ad selection}

The five ads used in the study were originally broadcast on U.S. television or appeared in U.S. magazines in 2002 and 2003. They were selected because of their broad exposure to U.S. audiences, the likelihood of large audiences under the MLPA, to represent multiple malt liquor 
and beer products, and to represent various violations of the 1997 U.S. Beer Institute Advertising and Marketing Code (Babor et al., 2008). Ad 1 (After College) shows an older African-American man describing his life after graduating from college to a younger African-American man. Both are drinking Colt 45. Ad 2 (Noise Compliant) shows a police officer investigating a noise complaint at a party where young persons are drinking Samuel Adams Light Beer. Ad 3 (Boat Trip) shows young adults lounging on a rapidly moving boat and passing bottles of Skyy Blue malt beverages to each other. Ad 4 (Poolroom Party) depicts three young men organizing a party at a pool hall after being denied entrance to a club. They are drinking Smirnoff Ice. Ad 5 (Stamp Collector) is a magazine ad that shows a man's forearm bearing six nightclub stamps and holding a Smirnoff Ice bottle. The tagline reads "4:06 A.M. We get past our sixth doorman of the evening." Violations included portraying alcohol in an irresponsible manner (Ads 3 and 5), promoting excessive alcohol consumption (Ad 5), including content that primarily appeals to individuals under the MLPA (Ad 1), and associating alcohol consumption with social, professional, education, athletic, or financial success (all ads). See Babor et al. (2008) for a complete description of the ad selection and other procedures.

\section{Categorizing vulnerable populations}

Heavy alcohol users were defined as any individual in AUDIT risk categories II or higher. These categories were collapsed due to the low prevalence of individuals in risk categories III and IV. These individuals were considered potentially vulnerable to alcohol marketing. Non-heavy alcohol users were defined as any individual in AUDIT risk category I.

\section{Data analysis}

Two-level, within-person, random-intercepts only hierarchical linear models with full maximum likelihood estimation were used to determine if the scores on the dependent variables varied as a function of alcohol use. The primary individual-level variable (AUDIT risk category) was dummy coded and entered into the model at Level-2 uncentered. The reference group was individuals in AUDIT risk category I.

All models were adjusted for age, race, ethnicity, sex, and parental alcohol use. Each covariate was dummy coded. Age was dichotomized into under the MLPA ( $<21$ years old) and over the MLPA ( $\geq 21$ years old). Race was defined as either White or non-White. Ethnicity was considered either Hispanic or non-Hispanic, and sex was classified as either male or female. Parental alcohol use history was categorized as having at least one parent be a heavy, problem, or former problem drinker during most of the time the participant was growing up or having neither parent be a heavy, problem, or former problem drinking during most of the time the participant was growing up. These variables were introduced as Level-2, uncentered covariates. There were no Level-1, or ad-level, variables.

$$
\begin{aligned}
Y_{i j}= & \pi_{o j}+e_{i j} \\
\pi_{o j}= & \beta_{00}+\beta_{01}(\mathrm{LPA})+\beta_{02}(\text { race })+\beta_{03}(\text { ethnicity }) \\
& +\beta_{04}(\mathrm{IAH})+\beta_{05}(\mathrm{PAH})+\beta_{06}(\text { gender })
\end{aligned}
$$

Intra-class correlations (ICCs) were calculated for each dependent variable using the null model. In order to remove the effects of older students who were recruited into the sample, a sensitivity analysis (Model 2) was conducted using only $18-24$ year olds $(n=257)$. Statistical analysis was performed using HLM for Windows Version 7.01 (Scientific Software International, Inc., Skokie, IL).

\section{Results}

In total, $49.7 \%$ of participants were under the MLPA, $60.7 \%$ were female, $56.4 \%$ were white, and $81.6 \%$ were non-Hispanic. The age range of participants was 18 to 79 years old. Overall, $42.3 \%$ of participants were categorized into AUDIT risk categories II (at-risk use), III (harmful use), or IV (referral for evaluation and treatment), and $73.0 \%$ had at least 1 parent positive for excessive alcohol use. Mean ad appeal across all ads was 2.9 $(\mathrm{SD}=1.1)$ with mean ad-specific ad appeal ranging from 2.3 to 3.3 (Table 1). Similar scores and ranges were seen for perceptions of excessive drinking and responsible drinking. Mean perceived alcohol consumption for the main character in the ads was 5.6 drinks per ad $(\mathrm{SD}=3.8)$, which met the CDC definition of binge drinking (CDC, 2016), with mean ad-specific alcohol consumption ranging from 2.7 to 9.2 drinks per ad.

In Model 1, ICC was ranged from 0.09 to 0.31 and all random effects were statistically significant $(p$ 's $<0.001)$ (Table 2). Individual alcohol use history was positively associated with ad appeal ( $\mathrm{p}<0.001)$, perceived responsible drinking $(\mathrm{p}=0.011)$, and total perceived alcohol consumption $(p=0.002)$. There were no significant differences in any of the dependent variables based on age, race, ethnicity, or parental alcohol history. Women were more likely to view alcohol consumption in the ads as excessive compared to men ( $p=0.002)$.

In Model 2, ICC was ranged from 0.08 to 0.30 and all random effects were statistically significant $(p$ 's $<0.001)$ (Table 3). Individual alcohol use history was positively related to ad appeal $(p=0.007)$ and total perceived alcohol consumption $(p=0.001)$. There were no significant differences in any of the dependent variables based on 
Table 1. Mean perceptions of each ad included in the study*

\begin{tabular}{lcccc}
\hline Ad & Appeal $^{* *}$ & Excessive drinking $^{* *}$ & Responsible drinking $^{* * *}$ & Alcohol consumption $^{* * *}$ \\
\hline 1 & $3.0(1.1)$ & $2.4(1.1)$ & $2.6(1.0)$ & $4.0(2.2)$ \\
2 & $2.3(1.0)$ & $2.0(1.0)$ & $3.7(0.9)$ & $2.7(2.2)$ \\
3 & $3.3(1.1)$ & $2.8(1.2)$ & $2.8(0.9)$ & $5.6(2.5)$ \\
4 & $3.2(1.1)$ & $3.4(1.2)$ & $2.5(1.0)$ & $6.7(3.4)$ \\
5 & $2.6(1.1)$ & $3.1(1.4)$ & $2.3(0.9)$ & $9.2(4.4)$ \\
Total & $2.9(1.1)$ & $2.7(1.3)$ & $2.8(1.0)$ & $5.6(3.8)$ \\
\hline
\end{tabular}

*Mean (SD); ${ }^{* *}$ Likert scale scores (possible range $=1-5$ ); ${ }^{* * *}$ number of drinks.

Table 2. Association between membership in a vulnerable population group and ad perceptions, full sample $(n=326)$.

\begin{tabular}{lccrr}
\hline & Ad appeal & Excessive drinking & Responsible drinking & Alcohol consumption \\
\hline ICC & 0.31 & 0.16 & 0.10 & 0.09 \\
Fixed effects & & & & \\
$\quad$ Intercept $\left(\beta_{00}\right)$ & $2.68(0.13)^{* * *}$ & $2.65(0.12)^{* * *}$ & $2.64(0.09)^{* * *}$ & $5.37(0.33)^{* * *}$ \\
$\quad$ Individual alcohol history $\left(\beta_{01}\right)$ & $0.33(0.09)^{* * *}$ & $-0.10(0.09)$ & $0.16(0.06)^{*}$ & $0.72(0.23)^{* *}$ \\
MLPA $\left(\beta_{02}\right)$ & $0.11(0.08)$ & $0.02(0.08)$ & $0.04(0.06)$ & $0.20(0.25)$ \\
Race $\left(\beta_{03}\right)$ & $-0.03(0.10)$ & $-0.13(0.10)$ & $-0.04(0.07)$ & $0.19(0.26)$ \\
Ethnicity $\left(\beta_{04}\right)$ & $0.15(0.12)$ & $0.03(0.12)$ & $0.02(0.09)$ & $-0.22(0.31)$ \\
Parental alcohol history $\left(\beta_{05}\right)$ & $-0.04(0.09)$ & $0.02(0.09)$ & $0.06(0.25)$ & $0.13(0.22)$ \\
$\quad$ Gender $\left(\beta_{06}\right)$ & $0.03(0.08)$ & $0.26(0.08)^{* *}$ & $0.06)$ & $1.10^{* * *}$ \\
Random effects & $0.36^{* * *}$ & $0.24^{* * *}$ & $0.09^{* * *}$ & \\
$\quad$ Intercept $(\tau)$ & & &
\end{tabular}

${ }^{*} p<0.05 ;{ }^{* *} p<0.01 ;{ }^{* *} p<0.001$; reference groups $=$ over the legal purchase age, White, non-Hispanic, AUDIT risk category I, neither parent with a history of excessive alcohol use, and male.

age, race, ethnicity, or parental alcohol history. Women were more likely to view alcohol consumption in the ads as excessive compared to men $(p<0.001)$.

\section{Discussion}

Among the sample of U.S. college students, heavy alcohol users perceived alcohol ads as more appealing, perceived greater alcohol consumption in the ads, and perceived high levels of consumption as "responsible," even though, on average, study participants perceived alcohol consumption in the ads to meet the definition of binge drinking $\left(\bar{X}_{\text {Drinks }}=5.6\right)$. Secondary findings indicate that men were less likely to view alcohol consumption in the ads as excessive, and that there were no significant differences in ad perceptions based on age.
The study results support the hypothesis that alcohol ad perceptions differ amongst individuals with a history of excessive alcohol use, and this finding is consistent with previous research. Greater reactivity to alcohol cues has been demonstrated in non-dependent binge drinkers compared to light drinkers (Petit, Kornreich, Verbanck, \& Campanella, 2013), and binge drinking has been associated with greater reactivity to alcohol cues and lower reactivity to non-alcohol cues (Petit, Kornreich, Dan, Verbanck, \& Campanella, 2014). Moreover, heavy drinkers may have difficulty disengaging from alcohol cues due to increased attentional bias towards the cue and a decreased ability to focus on non-alcohol related stimuli (Storkmark, Field, Hugdahl, \& Horowitz, 1997). However, the current study differs from previous cueexposure studies due to the additional context an alcohol

Table 3. Association between membership in a vulnerable population group and ad perceptions, 18-24 year olds only ( $n=257)$.

\begin{tabular}{|c|c|c|c|c|}
\hline & Ad appeal & Excessive drinking & Responsible drinking & Alcohol consumption \\
\hline ICC & 0.30 & 0.16 & 0.11 & 0.08 \\
\hline \multicolumn{5}{|l|}{ Fixed effects } \\
\hline Intercept $\left(\beta_{00}\right)$ & $2.75(0.15)^{* * *}$ & $2.53(0.15)^{* * *}$ & $2.63(0.11)^{* * *}$ & $5.22(0.40)^{* * *}$ \\
\hline Individual alcohol history $\left(\beta_{01}\right)$ & $0.26(0.10)^{* *}$ & $-0.03(0.09)$ & $0.13(0.07)$ & $0.85(0.26)^{* *}$ \\
\hline $\operatorname{MLPA}\left(\beta_{02}\right)$ & $0.03(0.10)$ & $0.08(0.10)$ & $0.04(0.08)$ & $0.22(0.26)$ \\
\hline Race $\left(\beta_{03}\right)$ & $-0.13(0.11)$ & $-0.04(0.11)$ & $-0.10(0.08)$ & $0.05(0.30)$ \\
\hline Ethnicity $\left(\beta_{04}\right)$ & $0.13(0.13)$ & $0.03(0.13)$ & $0.02(0.09)$ & $0.03(0.34)$ \\
\hline Parental alcohol history $\left(\beta_{05}\right)$ & $-0.02(0.10)$ & $-0.07(0.10)$ & $0.05(0.08)$ & $-0.33(0.28)$ \\
\hline Gender $\left(\beta_{06}\right)$ & $0.17(0.09)$ & $0.34(0.09)^{* * *}$ & $0.12(0.07)$ & $0.20(0.25)$ \\
\hline \multicolumn{5}{|l|}{ Random effects } \\
\hline Intercept $(\tau)$ & $0.35^{* * *}$ & $0.23^{* * *}$ & $0.10^{* * *}$ & $1.04^{* * *}$ \\
\hline
\end{tabular}


advertisement provides to the alcohol product stimulus, and depictions of parties and scenes of camaraderie may amplify the effect of the physical appearance of alcohol. Indeed, several studies have demonstrated that heavy drinkers have greater reactivity to drinking contexts, such as parties, than light drinkers (Lee et al., 2006), and others have reported that alcohol dependence symptoms are associated with perceived drinking in alcohol ads (Proctor et al., 2005). Depictions of drinking occasions can also stimulate alcohol cravings in the absence of direct alcohol cues (Mason, Light, Escher, \& Drobes, 2008). Furthermore, increased activation of neural pathways associated with visual attention, memory, motivation, and habit has been observed in heavy drinkers after exposure to alcohol cues, and pathway activation was associated with increased alcohol cravings (Dager et al., 2013; Tapert, Brown, Baratta, \& Brown, 2004).

Heavy alcohol users perceived greater alcohol consumption in the ads but did not perceive such consumption as excessive. This lack of association may have both a theoretical and a practical explanation. The non-relationship may be explained using the Theory of Planned Behavior (TPB). TPB posits that behavioral intention and behavior is due, in part, by subjective norms and normative beliefs (Ajzen, 1991). Regarding perceptions of alcohol advertisements, if an individual was exposed to an alcogenic environment that either accepted or rewarded high rates of alcohol consumption, higher perceived alcohol consumption would be viewed as normal. Practically, there could have been a difference in perceptions of excessive alcohol consumption, but the study was underpowered to detect such a difference.

Expectancy theory could explain the association between alcohol use and ad appeal. Expectancy theory suggests that individuals who have greater positive alcohol expectancies and fewer negative alcohol expectancies are more likely to consume excessive quantities of alcohol (Hays, 1985). These perceptions may be reinforced when alcohol ads portray social, financial, physical, and sexual benefits of alcohol use and little to no negative consequences. Indeed, perceptions of alcohol ads have predicted positive alcohol expectancies in samples of youths, teens and young adults (Fleming, Thorson, \& Atkin, 2004). The application of expectancy theory assumes that exposure to alcohol marketing is causally related to alcohol initiation or increased consumption among current drinkers, which has been demonstrated by others (Anderson et al., 2009; Jernigan et al., 2017; Smith \& Foxcroft, 2009).

Interestingly, men were less likely to perceive alcohol consumption in the ads as excessive compared to women, and the magnitude of this effect was larger in the sensitivity analysis. Coupled with findings that men, especially young men, are disproportionately affected by the negative consequences of heavy alcohol consumption (U.S. Department of Health and Human Services, 2007), the results suggest that men could be considered a potentially vulnerable group, pending additional research. Additionally, there were no significant differences in ad perceptions between participants above and below the MLPA, which may be more indicative of the inability to construct an advertisement that is appealing to a 21 year old but is not appealing to a 20 year old.

The current findings augment recent research on the cognitive impacts of alcohol advertising. In a randomized experiment involving 373 adults, positive implicit attitudes towards alcohol were increased, and negative implicit attitudes were decreased, after exposure to alcohol advertisements (Brown et al., 2016). In a similar experiment involving only heavy drinking young adults, 18-25 years old, exposure to alcohol advertisements was associated with high mean pleasure and arousal scores as well as decreased alcohol avoidance bias (Stautz et al., 2017). The results of a meta-analysis support these findings (Stautz et al., 2016). Furthermore, evidence suggests that alcohol consumers preferentially pay more attention to alcohol branding on alcoholic beverage labels than warning labels, even after motivation to reduce drinking was increased through a brief intervention (Kersbergen \& Field, 2017).

A two-part test to determine if a population group should be considered vulnerable to alcohol marketing has been proposed (Babor, Robaina, Noel, \& Ritson, 2017). To meet this definition of "vulnerable," a group needs to be particularly vulnerable to the health effects of alcohol use and to alcohol marketing. Given the significant short and long-term consequences of heavy alcohol use (CDC, 2016), heavy drinkers likely satisfy the first requirement of the test. The results described here and elsewhere suggest that heavy drinkers may also satisfy the second requirement.

The current codes used in self-regulatory alcohol marketing systems contain specific provisions preventing the promotion of excessive alcohol consumption (IARD, 2011). However, these provisions may be insufficient if heavy alcohol users perceive depictions of binge drinking even when moderate consumption is depicted and perceive such depictions as responsible, as reported here. If the current system of self-regulatory alcohol marketing is to continue, the findings indicate that two significant changes are needed to improve the protection of vulnerable groups. First, self-regulatory alcohol marketing codes should include a specific definition of vulnerability, such as that described by Babor et al. (2017), and alcohol marketers should be banned from using content that may appeal to groups that meet this definition. Second, stronger implementation and enforcement of existing codes is needed. ICAP's Guiding Principles do not 
provide alcohol producers with a system of preproduction review of alcohol ads nor do they specify any penalties for non-compliance. Currently, the only method of removing non-compliant ads from the public domain is through a complaint process that has been called ineffective because ads have already been viewed by the public and potentially biased (Noel \& Babor, 2017). For example, complaints against beer advertisements can be submitted to the U.S. Beer Institute's Code Compliance Review Board (CCRB), but each members of the CCRB has significant conflicts of interest that make independent ad adjudications unlikely. An independent pre-clearance would ensure that the letter and spirit of self-regulated alcohol marketing codes are adhered to prior to exposure to potentially vulnerable populations.

\section{Limitations}

There are several limitations to this study. This is a secondary analysis of cross-sectional data. As such, causal pathways cannot be determined, and the findings may be better interpreted as a first step towards expanding the definition of vulnerability in relation to alcohol marketing regulation rather than a definitive answer. The ads were not selected at random but meant to be representative of ads that violated self-regulated alcohol marketing codes. It is possible that ad perceptions may differ when using ads that are compliant with the codes, which may limit the generalizability of the findings. The ads were also broadcast and published in the early 2000s, and it is possible that content of advertising has changed over time. However, content analyses have revealed that similar themes have been used in alcohol advertisements since the 1980s and that violations of self-regulatory marketing codes are common, with violations rates commonly exceedingly $75 \%$ (Noel et al., 2017). Moreover, ads today are viewed on multiple media platforms, and the findings may not be applicable to media other than television. Ads were shown to participants in the same order, and order effects may partially explain the result. Additionally, later appearing ads may have had a lesser impact on the results than earlier appearing ads due to respondent fatigue.

Participants were recruited from only two colleges and universities, and the sample may not be representative of the U.S. college population. For example, there was a high prevalence of individual and parental alcohol use problems, which may be due to recruiting at a community college (Chen \& Paschall, 2003; Sheffield, Darkes, Del Boca, \& Goldman, 2005). Moreover, the use of a convenience sample of students may not be representative of all young people in the U.S. These limitations of the sample may further limit the generalizability of the findings. The study relied on self-report for all measurements, and individual responses could not be verified. It is possible that participants may have underreported undesirable characteristics (e.g. alcohol use, parental alcohol use, and ad appeal) and overreported desirable responses (e.g. perceived excessive drinking) due to social desirability bias. Because of the high prevalence of reported harmful alcohol use and excessive parental alcohol use, such biases likely only have minimal impact on the results.

\section{Conclusions}

Excessive drinking was associated with perceiving alcohol ads to have greater appeal and perceiving greater alcohol consumption in the ads. Heavy alcohol users also perceived high levels of alcohol consumption as responsible. These results indicate that heavy alcohol users may be a vulnerable population and marketing codes should be revised to include protections for these individuals. Future research is needed to confirm these findings and investigate potential mechanisms of action.

\section{Declaration of interest}

The authors report no conflicts of interest.

\section{Funding}

This work was supported by the National Institute on Alcohol Abuse and Alcoholism [Grant number R21 AA013530]; and the Beever Trust Fund. The sources of funding had no further role in study design; in the collection, analysis and interpretation of data; in the writing of the report; or in the decision to submit the paper for publication.

\section{References}

Ajzen, I. (1991). The theory of planned behavior. Organizational Behavior and Human Decision Processes, 50, 179-121. doi:10.1016/0749-5978(91)90020-T.

Anderson, P., de Bruijn, A., Angus, K., Gordon, R., \& Hastings, G. (2009). Impact of alcohol advertising and media exposure on adolescent alcohol use: A systematic review of longitudinal studies. Alcohol and Alcoholism, 44(3), 229-243. doi:10.1093/alcalc/agn115.

Babor, T. F., Robaina, K., Noel, J. K., \& Ritson, E. B. (2017). Vulnerability to alcohol-related problems: A policy brief with implications for the regulation of alcohol marketing. Addiction, 112(S1), 94-101. doi:10.1111/add.13626.

Babor, T. F., Xuan, Z., \& Proctor, D. (2008). Reliability of a rating procedure to monitor industry selfregulation codes governing alcohol advertising content. Journal of Studies on Alcohol and Drugs, 69, 235-242. doi:10.15288/jsad.2008.69.235.

Bar-Haim, Y., Lamy, D., Pergamin, L., Bakermans-Kranenburg, M. J., \& van IJzendoorn, M. H. (2007). Threat-related attentional bias in anxious and nonanxious individuals: A 
meta-analytic study. Psychological Bulletin, 133(1), 1-24. doi:10.1037/0033-2909.133.1.1.

Brown, K. G., Stautz, K., Hollands, G. J., Winpenny, E. M., \& Marteau, T. M. (2016). The cognitive and behavioural impact of alcohol promoting and alcohol warning advertisements: An experimental study. Alcohol and Alcoholism, 51(3), 354-362. doi:10.1093/alcalc/agv104.

Centers for Disease Control and Prevention. (2016). Fact Sheets - Alcohol use and your health. Retrieved from https://www.cdc.gov/alcohol/fact-sheets/alcohol-use.htm

Chen, M., \& Paschall, M. (2003). Malt liquor use, heavy/problem drinking and other problem behaviors in a sample of community college students. Journal of Studies on Alcohol, 64(6), 835-842. doi:10.15288/jsa.2003.64.835.

Cox, W., Pothos, E., \& Hosier, S. (2007). Cognitive-motivational predictors of excessive drinkers' success in changing. Psychopharmacology, 192, 499-510. doi:10.1007/s00213-0070736-9.

Dager, A. D., Anderson, B. M., Stevens, M. C., Pulido, C., Rosen, R., Jiantonio-Kelly, R. E., ... Pearlson, G. D. (2013). Influence of alcohol use and family history of alcoholism on neural response to alcohol cues in college drinkers. Alcoholism: Clinical and Experimental Research, 37(S1), E161E171. doi:10.1111/j.1530-0277.2012.01879.x.

Field, M., \& Cox, W. M. (2008). Attentional bias in addictive behaviors: A review of its development, causes, and consequences. Drug and Alcohol Dependence, 97(1-2), 1-20. doi:10.1016/j.drugalcdep.2008.03.030.

Field, M., Duka, T., Eastwood, B., Child, R., Santarcangelo, M., \& Gayton, M. (2007). Experimental manipulation of attentional biases in heavy drinkers: Do the effects generalise? Psychopharmacology, 192, 593-608. doi:10.1007/s00213007-0760-9.

Field, M., Werthmann, J., Franken, I., Hofmann, W., Hogarth, L., \& Roefs, A. (2016). The role of attentional bias in obesity and addiction. Health Psychology, 35(8), 767-780. doi:10.1037/hea0000405.

Fleming, K., Thorson, E., \& Atkin, C. K. (2004). Alcohol advertising exposure and perceptions: Links with alcohol expectancies and intentions to drink or drinking in underaged youth and young adults. Journal of Health Communication, 9(1), 3-29. doi:10.1080/10810730490271665.

Hays, R. (1985). An integrated value-expectancy theory of alcohol and other drug use. British Journal of Addiction, 80, 379384. doi:10.1111/j.1360-0443.1985.tb03009.x.

International Alliance for Responsible Drinking. (2011). Guiding principles: Self-regulation of marketing communications for beverage alcohol. Retrieved from http://www.iard. org/wp-content/uploads/2016/01/Guiding-Principles.pdf

Jernigan, D., Noel, J., Landon, J., Thornton, N., \& Lobstein, T. (2017). Alcohol marketing and youth alcohol consumption: A systematic review of longitudinal studies published since 2008. Addiction, 112(Suppl 1), 7-20. doi:10.1111/add.13591.

Kersbergen, I., \& Field, M. (2017). Alcohol consumers' attention to warning labels and brand information on alcohol packaging: Findings from cross-sectional and experimental studies. BMC Public Health, 17, 123. doi:10.1186/s12889017-4055-8.

Lee, E., Namkoong, K., Lee, C. H., An, S. K., \& Lee, B. O. (2006). Differences of photographs inducing craving between alcoholics and non-alcoholics. Yonsei Medical Journal, 47(4), 491-497. doi:10.3349/ymj.2006.47.4.491.
Mason, B., Light, J., Escher, T., \& Drobes, D. (2008). Effect of positive and negative affective stimuli and beverage cues on measures of craving in non treatment-seeking alcoholics. Psychopharmacology, 200(1), 141-150. doi:10.1007/s00213008-1192-x.

Noel, J. K., \& Babor, T. F. (2017). Does industry self-regulation protect young persons from exposure to alcohol marketing? A review of compliance and complaint studies. Addiction, 112(Suppl 1), 51-56. doi:10.1111/add.13432.

Noel, J. K., Babor, T. F., \& Robaina, K. (2017). Industry selfregulation of alcohol marketing: A systematic review of content and exposure research. Addiction, 112(Suppl 1), 28-50. doi:10.1111/add.13410.

Petit, G., Kornreich, C., Dan, B., Verbanck, P., \& Campanella, S. (2014). Electrophysiological correlates of alcohol- and non-alcohol-related stimuli processing in binge drinkers: A follow-up study. Journal of Psychopharmacology, 28(11), 1041-1052. doi:10.1177/0269881114545663.

Petit, G., Kornreich, C., Verbanck, P., \& Campanella, S. (2013). Gender differences in reactivity to alcohol cues in binge drinkers: A preliminary assessment of eventrelated potentials. Psychiatry Research, 209(3), 494-503. doi:10.1016/j.psychres.2013.04.005.

Proctor, D. C., Babor, T. F., \& Xuan, Z. (2005). Effects of cautionary messages and vulnerability factors on viewers' perceptions of alcohol advertisements. Journal of Studies on Alcohol, 66(5), 648-657. doi:10.15288/jsa.2005.66.648.

Saunders, J., Aasland, O., Babor, T., de la Fuente, J., \& Grant, M. (1993). Development of the Alcohol Use Disorders Identification Test (AUDIT): WHO collaborative project on early detection of persons with harmful alcohol consumption-II. Addiction, 88, 791-804. doi:10.1111/j.1360-0443.1993.tb02093.x.

Sheffield, F., Darkes, J., Del Boca, F., \& Goldman, M. (2005). Binge drinking and alcohol-related problems among community college students: Implications for prevention policy. Journal of American College Health, 54, 137-141. doi:10.3200/JACH.54.3.137-142.

Smith, L. A., \& Foxcroft, D. R. (2009). The effect of alcohol advertising, marketing and portrayal on drinking behaviour in young people: Systematic review of prospective cohort studies. BMC Public Health, 9, 51. doi:10.1186/1471-24589-51.

Stautz, K., Brown, K. G., King, S. E., Shemilt, I., \& Marteau, T. M. (2016). Immediate effects of alcohol marketing communications and media portrayals on consumption and cognition: a systematic review and meta-analysis of experimental studies. BMC Public Health, 16, 465. doi:10.1186/s12889016-3116-8.

Stautz, K., Frings, D., Albery, I. P., Moss, A. C., \& Marteau, T. M. (2017). Impact of alcohol-promoting and alcohol-warning advertisements on alcohol consumption, affect, and implicit cognition in heavy-drinking young adults: A laboratory-based randomized controlled trial. British Journal of Health Psychology, 22(1), 128-150. doi: 10.1111/bjhp.12221.

Storkmark, K. M., Field, N. P., Hugdahl, K., \& Horowitz, M. (1997). Selective processing of visual alcohol cues in abstinent alcoholics: An approach-avoidance conflict? Addictive Behaviors, 22(4), 509-519. doi:10.1016/S03064603(96)00051-2.

Tapert, S. F., Brown, G. G., Baratta, M. V., \& Brown, S. A. (2004). fMRI BOLD response to alcohol stimuli in alcohol 
dependent young women. Addictive Behaviors, 29(1), 3350. doi:10.1016/j.addbeh.2003.07.003.

U.S. Department of Health and Human Services. (2007). The surgeon general's call to action to prevent and reduce underage drinking. Rockville, MD: U.S. Department of Health and Human Services, Office of the Surgeon General. Retrieved from
http://www.ncbi.nlm.nih.gov/books/NBK44360/pdf/Book shelf_NBK44360.pdf

Vendrame, A., Pinsky, I., Faria, R., \& Silva, R. (2009). Brazilian teenagers and beer advertising: Relationship between exposure, positive response, and alcohol consumption. Cadernos de Saúde Pública, 25(2), 359-365. doi:10.1590/S0102311X2009000200014. 